\title{
ORT.24 - Immunossupression murine model to study antiviral resistance emergence during Influenza A infection
}

\author{
Thayssa Keren Santos da Silva ${ }^{1 *}$. \\ 1 Fiocruz - Fundação Oswaldo Cruz.
}

Introduction: Influenza virus infection is a major cause of worldwide morbidity and mortality, affecting thousands of people annually. Severe cases are treated with viral Neuraminidase inhibitors like Tamiflu (Oseltamivir). However, high genetic variation could lead to emergence of antiviral resistant strains, limiting the treatment effectiveness. Antiviral-resistant viruses may arise spontaneously, by inappropriate antiviral use or by prolonged viral shedding observed in immunosuppressed patients.

Objective: Our overall objective was to establish a murine model to study the emergence of influenza A virus resistant to Tamiflu and to investigate the effect of an immunosuppressive treatment with Dexamethasone and/or Cyclophosphamide in combination with a subtherapeutic dose of Tamiflu on immunopathology and emergence of resistance.

Methodology: C57/BL6 mice infected with Influenza A/PR/8/34 H1N1 (PR8) were treated with Tamiflu in different doses $-0.1,1$ or $10 \mathrm{mg} / \mathrm{kg}$, or vehicle - after two days of infection, and were monitored for weight loss and lethality for 21 days. Alternatively, mice were euthanized after 7 days of infection to perform bronchoalveolar lavage (BAL), viral isolation, titration and Oseltamivir resistance test by Neuraminidase activity assay (NA-Star) and inflammation. In a new assay, infected mice were treated with dexamethasone before infection or 2 days after infection, alone or in combination with Tamiflu ( $1 \mathrm{mg} / \mathrm{kg})$. Alternatively, dexamethasone from day -1 to day 10 was used in combination with cyclophosphamide at days -1 and 5 . After 7 or 10 days of infection, mice were euthanized and the same analyzes were performed.

Results: Treatment with the $10 \mathrm{mg} / \mathrm{kg}$ of Tamiflu, but not the other doses, reduced lethality, weight loss and viral titers in the lungs compared to vehicle. No reduced susceptibility to Oseltamivir were found in different treatments when compared vehicle or PR8. 10mg/kg of Tamiflu reduced total leukocytes, neutrophils in BAL and lung and IFN- $\gamma$ in BAL. The dose of $1 \mathrm{mg} / \mathrm{kg}$ partially reduced lethality rates and inflammation. Dexamethasone induced a slight immunosuppression. Only the group post-treated with dexamethasone plus Tamiflu was protected from lethality, weight loss and inflammation compared to vehicle group. Viruses isolated from all groups at day 7 did not show reduced susceptibility to oseltamivir. After ten days of infection, no viruses were isolated. A new immunosuppressive protocol using a combination of Dexamethasone and Cyclophosphamide led to a high level of immunosuppression and viral persistence until the tenth the of infection, whereas the vehicle group did not present any virus left on the lungs. Further analysis regarding emergence of virus with reduced susceptibility to oseltamivir will be performed using the Dexamethasone and Cyclophosphamide treatment.

Conclusion: Subtherapeutic treatment with Tamiflu does not reduce susceptibility to oseltamivir. Dexamethasone treatment in combination with Tamiflu might improve influenza manifestations without favoring viral resistance. Immunosuppression conferred by Cyclophosphamide leads to viral persistence.

Keywords: Influenza A; Resistance; Immunossuppression 